\title{
Le soleil, les brigands et la charrette
}

\section{Françoise Létoublon}

\section{OpenEdition}

\author{
Journals
}

Édition électronique

URL : http://journals.openedition.org/recherchestravaux/265

DOI : 10.4000/recherchestravaux.265

ISSN : 1969-6434

\section{Éditeur}

UGA Éditions/Université Grenoble Alpes

\section{Édition imprimée}

Date de publication : 30 mai 2005

Pagination : 93-101

ISBN : 0151-1874

ISSN : 0151-1874

\section{Référence électronique}

Françoise Létoublon, « Le soleil, les brigands et la charrette », Recherches \& Travaux [En ligne], $67 \mid$ 2005, mis en ligne le 30 septembre 2008, consulté le 08 septembre 2020. URL : http://

journals.openedition.org/recherchestravaux/265; DOI : https://doi.org/10.4000/recherchestravaux 265

(c) Recherches \& Travaux 
Françoise LÉTOUBLON

Université Stendhal - Grenoble 3

\section{Le soleil, les brigands et la charrette}

Le soleil avait achevé plus de la moitié de sa course et son char, ayant attrapé le penchant du monde, roulait plus vite qu'il ne voulait. Si ses chevaux eussent voulu profiter de la pente du chemin, ils eussent achevé ce qui restait du jour en moins d'un demi-quart d'heure; mais, au lieu de tirer de toute leur force, ils ne s'amusaient qu'à faire des courbettes, respirant un air marin qui les faisait hennir et les avertissait que la mer était proche, où l'on dit que leur maître se couche toutes les nuits. Pour parler plus humainement et plus intelligiblement, il était entre cinq et six heures quand une charrette entra dans les halles du Mans. ${ }^{I}$

Le jour ne faisoit gueres que commencer à poindre $\&$ le soleil à rayer sur les cimes des montagnes, quand il se trouva une troupe d'hommes armés $\&$ embastonnés à la façon des brigans au-dessus du mont qui s'élève le long de l'une des bouches du Nil, que l'on appelle Héracléotique, lesquels s'arrestèrent illec un peu pour couvrir de l'œil la mer qui bat le pied de la montagne. ${ }^{2}$

La comparaison est claire, et si j'ose dire, lumineuse: le soleil se lève dans un cas, il se couche dans l'autre, plongeant dans les deux cas le lecteur au milieu d'un long périple dont les rebondissements vont le tenir en haleine - du moins l'auteur le souhaite-t-il - pendant longtemps. Le roman commence.

Scarron parodie dans l'incipit du Roman comique celui des Éthiopiques, le roman le plus réussi que l'Antiquité nous ait légué, celui que la célèbre traduction d'Amyot, avant Daphnis et Chloé, a rendu célèbre en Europe et dont elle a fait une sorte de modèle pour la littérature et l'iconographie. Scarron choisit ce texte à dessein, c'est le début de roman le plus original de la petite série de cinq romans grecs que nous connaissons. Il transpose dans un registre burlesque et trivial les aventures de Théagène et de Chariclée: le navire à l'ancre dans une baie que voient les brigands d'Héliodore devient une charrette, il est

I. Scarron, Le Roman comique, I, I.

2. Histoire Aethiopique de Héliodorus contenant dix livres, traitant des loyales \& pudiques amours de Theagenes thessalien et Chariclea Aethiopienne, traduite du grec en françois [par J. Amyot], éd. de Lyon, chez J. Huguetan, I589, revue et corrigée d'après l'éd. originale. 
vrai aussi lourdement chargée que le navire. Le cadre exotique du delta du Nil infesté de bandes de brigands est remplacé par la banalité triviale d'une ville française de province, Le Mans.

La cause est entendue: on pourrait s'arrêter là, s'en tenir à la constatation de cette relation d'imitation par le Roman comique du roman attribué à Héliodore en remarquant que la parodie dilate le texte imité en développant le thème du char du soleil qui ne figure pas dans le modèle. Scarron imiterait dans le genre romanesque le plus «idéaliste» des romans de l'Antiquités en tournant en dérision à travers sa première phrase ses procédés et la crédibilité d'une fiction qu'appréciaient tant ses lecteurs européens, comme l'attestent Shakespeare et Cervantes, pour nous en tenir aux plus grands génies de la littérature.

Pourtant, puisque cette parodie très précise ne semble guère avoir attiré l'attention des critiques jusqu'à présent ${ }^{4}$, il vaut peut-être la peine, en hommage à Jean Serroy, de regarder ces textes d'un peu plus près.

Chez Héliodore, la scène d'ouverture énigmatique est vue par des brigands, qui seront d'ailleurs chassés par une deuxième bande de brigands: les comédiens en voyage de Scarron ne sont donc pas les premiers "anti-héros" de la tradition romanesque. Bien que le roman d'Héliodore ne puisse être considéré dans son ensemble comme parodique, alors que celui d'Achille Tatius pourrait à mon sens donner lieu à une telle interprétation, soutenue par un critique il y a déjà bien longtemps, il me semble que le début des Éthiopiques, montrant la scène de massacre sans vainqueurs visibles à travers les yeux de brigands stupéfaits par ce spectacle, et poursuivant avec la mise en fuite de cette première bande par une autre, est d'un ton pour le moins mitigé: pas comique certes, mais pas tout à fait sérieux non plus. Le modèle auctorial, dont Scarron semble se moquer, se moquait peut-être lui-même des topoi du début de roman, qui voulaient la mise en scène d'un beau jeune homme et d'une belle jeune fille destinés à tomber amoureux l'un de l'autre dès le "premier regards" et à chercher tout au long du roman à se réunir en dépit des obstacles rencontrés sur le chemin de l'aventure, souvent suscités justement par les brigands et pirates dont ce début pourrait se moquer.

3. Voir le titre donné par Amyot à l'édition de sa traduction, n’en déplaise à la verve de l'abbé Prévost qui s'est plu à voir en la chaste Chariclée une dévergondée...

4. Rien dans les deux éditions du Roman comique que j'ai pu consulter, celle de É. Magne en Classiques Garnier (1955) qui s'est intéressé surtout aux «clefs» du roman, celle de J. Serroy chez Folio-Gallimard (1985), beaucoup plus intéressé par les aspects proprement littéraires du roman.

5. Voir J. Rousset, Leurs yeux se rencontrèrent. La scène de première vue dans le roman, Paris, J. Corti, I98I. 
Quant au thème des comédiens itinérants ${ }^{6}$, il existe aussi chez Héliodore (et dans plusieurs autres romans du corpus antique) où les héros rencontrent des troupes de théâtre, occasion de plusieurs de ces fausses morts dont le genre était avide - et qu'il a léguées au roman baroque. Dans les Éthiopiques, Chariclée devient comédienne travestie avec son mentor Calasiris, et Théagène se laisse d'ailleurs prendre au jeu de l'illusion théâtrale quand, sous son déguisement, elle le retrouve miraculeusement et se jette à son cou, d'où la fameuse scène du «soufflet» qui a fourni à l'abbé Prévost sa vertueuse diatribe contre cette pure héroïne que selon moi, il affecte de prendre pour le contraire de ce qu'elle est.

Revenons à la scène initiale des deux romans: après la première phrase ampoulée sur le moment de la course du soleil, dont la grandiloquence est bien moindre d'ailleurs chez Héliodore que chez Scarron, apparaissent chez l'un le navire chargé de richesses, mais apparemment vide de passagers, chez l'autre la charrette chargée de tout le nécessaire à une troupe de théâtre. Puis, dans les deux cas aussi, une jeune fille mystérieuse, qui pourrait être une déesse pour les brigands d'Héliodore, sur laquelle Scarron passe plus rapidement non sans remarquer son accoutrement un peu schizophrène:

La charrette était pleine de coffres, de malles et de gros paquets de toiles peintes qui faisaient comme une pyramide au haut de laquelle paraissait une demoiselle habillée moitié ville, moitié campagne.

Chez Héliodore, on comprendra plus tard que Chariclée, notre héroïne, porte dans cette scène initiale son costume d'Artémis, qu'elle transporte partout avec elle en même temps que les fameux symbola, les objets de reconnaissance qui lui serviront à la fin du roman à prouver à ses parents, le roi et la reine d'Éthiopie, qu'elle est bien leur fille abandonnée à la naissance à cause de sa peau blanche, imitée de celle d'Andromède dans le portrait de famille que regardait sa mère dans la chambre royale au moment de sa conception: comédienne, Chariclée ne l'est pas vraiment, mais son identité est constamment travestie, suivant le modèle homérique d'Ulysse dans l'Odyssée, et son travestissement symbolique en Artémis peut facilement dégénérer dans une réduction parodique du roman en oripeaux de théâtre.

Pour compléter l'examen en parallèle de la scène initiale du roman comme ouverture d'une pièce de théâtre, il reste à faire paraitre le jeune homme, le jeune premier: Scarron enchaîne très vite après sa phrase rapide sur la jeune

6. Thème qui se retrouve, à la suite de divers avatars tels que le Capitaine Fracasse, dans le film qui a fait connaitre Angelopoulos en France, O Thiassos («La troupe», 1974), justement traduit dans sa version française par Le Voyage des comédiens. 
fille au vêtement double: la description du jeune homme est beaucoup plus longue, et l'important est qu'il est blessé comme Théagène l'est:

Un jeune homme, aussi pauvre d'habits que riche de mine, marchait à côté de la charrette. Il avait un grand emplâtre sur le visage, qui couvrait un œil et la moitié de la joue, et portait un grand fusil sur son épaule [...]

J'abrège. Mais une phrase me semble un bon indice du fait que La Caverne est un avatar de Chariclée comme Le Destin celui de Théagène, celle qui descend de la tête jusqu'aux pieds de ce jeune homme:

Il portait des chausses trouées à bas d'attaches, comme celles des comédiens quand ils représentent un héros de l'antiquité, et il avait, au lieu de souliers, des brodequins à l'antique que les boues avaient gâtés jusqu'à la cheville du pied.

Mon hypothèse devient plus claire: pour la résumer en la poussant à sa limite, le Roman comique, c'est l'histoire de Théagène et de Chariclée, venue de l'Antiquité et tombée dans le ruisseau, couverte de boue et de dérision par la transformation des nobles personnages d'Héliodore, comédiens par obligation de se cacher dans les immenses dangers qui les menacent, en comédiens professionnels, travestis pour gagner leur vie et jusque dans leurs noms, dont il faudra reparler.

On pourrait m'objecter que ce n'est pas le seul exemple d'imitation de la première phrase des Éthiopiques; Gerald Sandy cite d'après Maurice Lever trois débuts de romans du XVII e siècle, sans donner de références:

- La nuict estoit desja bien avancée et la Lune pour lors bien esloignée du bel astre qui lui donne la lumière $[\ldots]$

- Déjà le Soleil commençoit à dorer $[\ldots]$

- Les ombres de la nuict n’avoient pas encore fait place à la lumière [...]

- La nuict n'avait pas encore estendu ses voiles, quand Climander [... $]^{7}$

Ce trait se trouve d'ailleurs déjà dans les Métamorphoses d'Apulée, au début du livre III :

L'Aurore venait juste, de son bras de rose, de lancer dans le ciel son char aux chevaux étincelants de phalères pourprées lorsque je fus arraché à la sécurité de mon repos et rendu au jour par la fin de la nuit. ${ }^{8}$

Et le début de ce chapitre des Métamorphoses nous reconduit aux incipit de chants de l'Iliades et à la fameuse «Aurore aux doigts de rose» d'Homère. Un

7. The Classical Heritage in France, Leiden, E. Brill, 2002.

8. P. Grimal, Romans grecs et latins, Paris, Gallimard (Bibliothèque de la Pléiade), I958, p. I82. P. Grimal, dans son introduction à Apulée (p. I44), dit s'être fortement «inspiré» de la traduction de P. Valette dans l'édition des Belles Lettres, CUF.

9. Tous les chants ne commencent pas avec une Aurore et les Aurores ne coïncident pas constamment avec les débuts de chants, mais on peut malgré tout supposer que cette coïncidence relativement fréquente a donné lieu à un topos littéraire. 
simple sondage dans la littérature italienne de la Renaissance le confirme: je trouve dans une traduction du XVIII ${ }^{e}$ siècle de la Jérusalem délivrée du Tasse due au Prince Lebrun ${ }^{\mathrm{IO}}$, au début du chant XX:

Déjà le soleil avait rappelé les mortels à leurs travaux; déjà son char, conduit par les Heures, avait mesuré une partie de sa carrière.

Même si ce passage nous fait sourire aujourd'hui, on ne saurait soupçonner le Tasse d'écriture parodique, pas plus que son traducteur français. Pourtant, sa phrase est pratiquement aussi développée que celle de Scarron. La parodie dans le Roman comique ne réside donc pas dans la première phrase par ellemême, mais dans le fait qu'elle est la première phrase d'un roman précisément intitulé Roman comique, où tous les titres de chapitres sont par euxmêmes des pièges parodiquement tendus au lecteur qu'ils obligent à réfléchir sur sa lecture et sur le mode d'écriture du récit ${ }^{\text {II }}$, et où la structure des personnages elle-même s'avère parodique de celle des Éthiopiques.

Nous avons laissé la jeune fille perchée sur sa pyramide et le jeune homme blessé à la tête et chaussé d'antiques chaussures cheminant à l'entrée de la ville du Mans. Reprenons le texte à la lumière de cette interprétation d'une parodie qui n'est peut-être pas systématique mais qui est structurée: on a remarqué en passant que la blessure du jeune homme à la tête chez Scarron rappelle étrangement la manière dont, sans savoir davantage qui il est ${ }^{12}$, on voit pour la première fois Théagène: il a été grièvement blessé et est inanimé, peut-être mort, la tête posée sur les genoux de la jeune fille. Ses premières paroles sont rassurantes mais il pourrait encore s'agir d'un bref retour à la conscience.

La pyramide sur une charrette chez Scarron elle-même pourrait n'être pas due au hasard: chez Héliodore, Chariclée est assise sur un rocher, et la scène se passe au pays des Pyramides...

Mais un troisième personnage, un vieillard, nous servira de preuve: il apparaît dans l'entrée de la troupe dans la ville du Mans chez Scarron:

IO. Mon édition date de 1862 mais reproduit la préface de l'édition de I774, dont elle précise la date.

II. C. Spencer, «Aux marges du texte; Le Roman comique: des titres à la page», Vers un Thesaurus informatisé: topique des ouvertures narratives avant I80o, Actes du quatrième colloque international SATOR, (Montpellier, 25-27 octobe 1990), Montpellier, Centre d'études du XVIII siècle, université Paul-Valéry, I99I, p. 245-255.

I2. Parmi les topoi de l'incipit romanesque semble se trouver la "fiche d'identité" des deux personnages dans les romans grecs, du degré zéro représenté par les Éphésiaques à l'élaboration littéraire subtile de Chariton. Mais Héliodore présente au contraire ses personnages comme une énigme: dans le paysage de massacre, de fin de tragédie, que perçoivent les brigands, la seule candidate pour le statut de jeune première ressemble à une divinité, et le seul candidat pour celui de jeune premier a l'air mort ou mourant... 
Un vieillard vêtu plus régulièrement, quoique très mal, marchait à côté de lui. Il portait sur les épaules une basse de viole et, parce qu'il se courbait un peu en marchant, on l'eût pris pour une grosse tortue qui marchait sur les jambes de derrière..$^{13}$

Dans les Éthiopiques, sa présentation est retardée au livre III4, où le premier qui le rencontre est Cnémon, un personnage secondaire du roman, à qui il sera amené à raconter son histoire, au livre non sans quelques occasions de la retarder et de la faire attendre. Comme il fait une prière dans laquelle il mentionne les deux noms de Théagène et de Chariclée, Cnémon, leur ami de fraîche date ${ }^{\mathrm{IS}}$, celui-ci, stupéfait demande comment il les connaît et c'est ce vieillard, un prêtre égyptien habillé à la grecque, qui va raconter l'histoire de Théagène et de Chariclée et comment la sienne propre s'y est trouvée mêlée.

Au livre VI, Théagène et Chariclée sont séparés, Chariclée décide de partir à sa recherche en compagnie du vieux Calasiris en se déguisant tous deux en mendiants pour éviter d'être reconnus par les brigands qui les ont déjà faits prisonniers: le long passage qui suit (I, II-I2) pourrait avoir inspiré la verve de Scarron:

Chariclée et Calasiris commencèrent par se transformer en mendiants, en utilisant des haillons qu'ils avaient préparés, ensuite Chariclée se barbouilla le visage avec de la suie et l'enduisit de boue, et elle se mit sur le visage un voile tout rapiécé et sale qui lui dissimulait un oeil en pendant de travers sur son front; sous le bras, elle avait une besace, apparemment pour y mettre des morceaux de viande et des croûtes, mais où, en réalité, se trouvait sa robe sacrée de Delphes et ses couronnes, ainsi que les joyaux exposés par sa mère en même temps qu'elle et les signes qui lui permettraient de se faire reconnaître. Calasiris avait roulé le carquois de Chariclée dans de vieilles peaux de moutons et il le portait comme un fardeau quelconque en travers de ses épaules; il avait détendu la corde de l'arc, qui, aussitôt, se redressa; il en fit un bâton sur lequel il s'appuya lourdement de ses deux mains, et, quand il rencontrait quelqu'un, il se courbait plus que ne l'y contraignait son âge et traînait une jambe, et même, il y avait des moments où Chariclée le conduisait par la main.

Lorsqu'ils eurent achevé de se déguiser, ils se plaisantèrent réciproquement en se disant, pour se taquiner, à quel point le personnage qu' ils jouaient leur allait bien, à l'un et à l'autre $[\ldots]$.

I3. L'incongruité de la comparaison est longuement commentée dans le texte, prenant plus de place à vrai dire que la description du vieillard: cela fait partie des stratégies narratives de Scarron. I4. Éth. II, 2I: « [...] lorsqu’il vit, sur la rive, un vieillard qui errait; il parcourait à grands pas, le long du fleuve, une bonne longueur, allant et venant, avec l'air de confier ses soucis à la rivière. Sa chevelure était disposée comme le sont celles des prêtres; elle était entièrement blanche. Son menton était recouvert d'une barbe épaisse et vénérable. Sa robe et son vêtement avaient l'air grecs".

I5. Il a fait leur connaissance à la suite de la scène du livre $\mathrm{I}^{\text {er }}$ dans laquelle les brigands qu'on appelle les «Pâtres du Nil» les ont faits prisonniers. Cnémon a raconté son histoire à Théagène et Chariclée, mais eux-mêmes, à cause de diverses péripéties dont la fausse mort de Chariclée, mais la vraie mort de Thisbé qui poursuivait Cnémon, n’ont pas eu le temps de raconter la leur. 
En trouvant l'origine chez Héliodore du vieillard qui accompagne les jeunes premiers de la troupe du Roman comique, nous avons rencontré aussi l'origine de l'emplâtre sur la figure et de la besace du jeune acteur de Scarron: si sa blessure lui vient bien de Théagène, son correspondant masculin dans le texte source, son emplâtre et sa besace pourraient venir de Chariclée travestie.

Les comédiens du Roman comique portent un nom pseudonyme, comme c'était l'usage au XVII siècle. Mais le choix de ces noms n'en est pas moins surprenant, comme le premier chapitre le dit d'ailleurs en les présentant, faisant d'ailleurs choir la jeune fille de son piédestal égyptien au vulgaire statut de poule:

Le jeune homme dont je viens de vous parler prit la parole, et, sans mettre les mains au turban, parce que, de l'une il tenait son fusil et de l'autre la garde de son épée, de peur qu'elle ne lui battît les jambes, lui dit qu'ils étaient Français de naissance, comédiens de profession; que son nom de théâtre était le Destin, celui de son vieil camarade, la Rancune, et celui de la demoiselle qui était juchée comme une poule au haut de leur bagage, la Caverne. Ce nom bizarre fit rire quelquesuns de la compagnie; sur quoi le jeune comédien ajouta que le nom de Caverne ne devait pas sembler plus étrange à ces hommes d'esprit que ceux de la Montagne, la Vallée, la Rose ou l'Épine.

La note Io de Jean Serroy à ce passage ${ }^{16}$ nous apprend que le dernier de ces noms est donné à des acteurs dans une pièce de Georges de Scudéry, ainsi que celui de Belle-Fleur dont celui de la Rose pourrait s'inspirer. Mais de la Caverne, point!

Chercher une origine à ce nom chez Héliodore pourrait sembler farfelu. Pourtant, une recherche antérieure sur le thème de la caverne chez Héliodore et Prévost, et la critique que le romancier Crébillon fit du thème des cavernes dans la tradition romanesque contre laquelle il se rebelle ${ }^{17}$ laissent penser qu' il y a peut-être là une forme de vérité. Chez Héliodore et chez Prévost, que l'inspiration soit passée de l'un à l'autre ou non, la caverne est un lieu de refuge, où certains des personnages vivent pendant quelque temps - pendant longtemps pour Cleveland; dans ces cavernes, les personnages ont du temps et ils le passent chez Héliodore à se raconter leurs histoires respectives, aux dépens de leur sommeil parfois; chez Prévost, Cleveland a eu dans Rumney-Hole les récits et lectures de sa mère, et les rencontres donnent lieu aussi à des récits.

16. Op. cit., p. 386.

17. Crébillon: "plus de morts imprévues et infiniment moins de souterrains», préface des Égarements du cour et de l'esprit, passage cité avec plus d'ampleur dans F. Létroublon, Les Lieux communs du roman, Leide, E. Brill, I993, p. 5 ; sur le thème de la caverne dans les romans grecs, id., p. 74-80; sur les cavernes de Prévost et leur relation possible aux Éthiopiques, F. Létoublon, "Les Leçons de Ténèbres", in L’Abbé Prévost au tournant du siècle, éd. par R. A. Francis et J. Mainil, Oxford, Voltaire Foundation, 2000, p. 26I-27I. 
Les récits s'y entrecroisent parfois de manière complexe chez les deux romanciers $^{18}$. J'en conclus que la caverne ou ses substituts est une image du cadre du récit et en devient parfois la métaphore: les deux cavernes transformées en lieu d'habitation dans les Éthiopiques et dans Cleveland sont en effet une sorte de dédale ou de labyrinthe dans lequel on peut s'égarer facilement, ou se méprendre sur l'identité de ceux que l'on rencontre (Thisbé prise pour Chariclée est tuée à sa place: le salut de Chariclée est donc dû à l'obscurité de la caverne qui a permis la méprise).

Scarron a certainement lu les Éthiopiques, comme Prévost après lui: la comédienne qui porte le nom de la Caverne raconte en effet son histoire à une de ses consœurs appelée l'Étoile au cours d'une nuit où elles sont amenées par les circonstances à dormir ensemble, et comme les personnages d'Héliodore, comme le feront encore ceux de Jacques le fataliste et de bien d'autres romans, elles se racontent des histoires pendant le temps réservé au sommeil. Comme chez Héliodore, le récit est interrompu par un événement imprévu, l'irruption d'un étrange personnage, un "fantôme lévrier»...

Mon hypothèse est que Scarron a fort bien senti l'importance du thème de la caverne dans le roman grec, et par là dans la tradition du roman qu'il parodie: la caverne est dans les Éthiopiques, ainsi d'ailleurs que dans Daphnis et Chloé - bien plus qu'un simple cadre. Elle joue un rôle dans l'intrigue du roman. D'où il a pu tirer l'idée d'en faire un rôle de théâtre dans son histoire de comédiens voyageurs. Il me semble que c'est le même procédé que l'on trouve dans la parodie par Shakespeare de l'histoire de Pyrame et Thisbé prise chez Ovide: la cloison qui sépare les amants ovidiens a une telle importance que les comédiens improvisés qui veulent mettre en scène cette aventure pour le mariage du roi dans le Songe d'une nuit d'été décident en effet qu'outre les personnages de Pyrame et de Thisbé, le Mur et le Clair de lune vont être incarnés par des acteurs: en prenant chez Héliodore les amours de Théagène et de Chariclée, Scarron aurait pu décider que la caverne dans laquelle se passe une partie de leurs aventures en Égypte recevrait le statut de personnage. Dans le chapitre VII, «L'aventure des brancards», les brancards destinés à chercher Mademoiselle de l'Étoile, comédienne de la troupe qu'un accident avait séparée de ses compagnons, montre comment ce processus peut advenir, comme Catherine Spencer l'a fait remarquer:

Je crois que tous les brancards de la province se sont donné rendez-vous pour une affaire d'importance ou pour un chapitre général, dit la Rancune, et je suis d'avis qu'ils commencent leur conférence.

I8. Sur la complexité des récits dans le roman grec et particulièrement chez Héliodore, voir aussi F. Létoublon, «Le roman grec, un océan ou un essaim d'histoires», Lalies, I3, p. 153-173. 
Mais la scène initiale du Roman comique, si elle imite celle des Éthiopiques en s'en moquant comme je le crois, lance le lecteur sur une fausse piste, ce qui n'étonnera personne: l'arrivée des comédiens dans la ville du Mans pourrait faire croire que le Destin et la Caverne sont les héros de l'histoire. Mais il faudra avancer dans la lecture pour comprendre que la Caverne, si j'ose dire, est une impasse, et que la vraie hérö̈ne n'est pas encore là : comme Thisbé est tuée à la place de Chariclée chez Héliodore, la Caverne juchée sur sa pyramide au début du roman était un leurre, on s'en apercevra seulement en apprenant que mademoiselle de l'Étoile, la «vraie» héroïne du roman, aimée du Destin, a été retardée en chemin par une douleur au pied, suscitant l'envoi d'un brancard et l'imbroglio de la rencontre des brancards en nombre au chapitre VII dont nous avons parlé ci-dessus: la complexité de la caverne chez Héliodore n'a pas fini de nous surprendre...

Usant d'une désinvolture dont Scarron fournit un charmant modèle, je ne donnerai pas de conclusion à ce texte, Au dédoublement de Scarron infirme en un jeune homme impatient de vivre, le Destin, et en un infirme ridicule, Ragotin, tel qu'il est analysé par Jean Serroy ${ }^{19}$, au jeu par lequel «le roman sert de revanche à la vie", il faut ajouter ce double-fond fourni par l'allusion à un double littéraire, au modèle idéal du roman transformé en aventure burlesque, à la boue de la modernité jetée sur la beauté des Chariclée et des Théagène venus de l'Antiquité. J. Serroy cite d'ailleurs à juste titre les Éthiopiques comme modèle des romans du temps de Scarron ${ }^{20}$ :

Un roman officiel s'est constitué ainsi, dont la structure est largement tributaire du modèle grec fourni par Héliodore dans les Éthiopiques. La multiplicité et la complexité des intrigues, les rebondissements d'une action toujours riche en péripéties, le rôle éminent joué par le hasard, la haute condition des personnages, la beauté de leurs prouesses, l'étrangeté des aventures qu'ils traversent, la place centrale tenue par les relations amoureuses, la noblesse du style qui en relate les développements, la complaisance prise à raconter des histoires qui s'emboîtent les unes dans les autres, ce qui entraîne des récits interminables: autant de caractères qui, à force d'être répétés, de D'Urfé aux Scudéry en passant par Gomberville ou La Calprenède, deviennent de véritables conventions.

Ces conventions que les romanciers de son temps ont empruntées à Héliodore, Scarron les reprend et en fait sa matière première pour les tourner en dérision et faire rire son lecteur dès les premières lignes.

19. Préface à son édition du Roman comique, 1985, p. 7-31, en particulier p. I2-I5.

20. Ibid., p. I5-I6. 